The Astrophysical Journal, 658:1188-1195, 2007 April 1

(C) 2007. The American Astronomical Society. All rights reserved. Printed in U.S.A.

\title{
HUBBLE SPACE TELESCOPE AND OPTICAL OBSERVATIONS OF THREE PULSATING ACCRETING WHITE DWARFS IN CATACLYSMIC VARIABLES ${ }^{1}$
}

\author{
Paula Szkody, ${ }^{2}$ Anjum Mukadam, ${ }^{2}$ Boris T. Gänsicke, ${ }^{3}$ Patrick A. Woudt, ${ }^{4}$ Jan-Erik Solheim, ${ }^{5}$ Atsuko Nitta, ${ }^{6}$ \\ Edward M. Sion, ${ }^{7}$ Brian Warner, ${ }^{4}$ D. K. Sahu, ${ }^{8}$ T. Prabhu, ${ }^{8}$ and Arne Henden ${ }^{9}$ \\ Received 2006 September 28; accepted 2006 December 13
}

\begin{abstract}
Ultraviolet observations using the Solar Blind Channel on the Hubble Space Telescope provide light curves and low-resolution spectra of three pulsating white dwarfs in the cataclysmic variables SDSS J013132.39-090122.3, SDSS J161033.64-010223.3, and SDSS J220553.98+115553.7. The UV light curves show enhanced pulsation amplitudes over those from simultaneous and previous optical photometry, while the UV-optical spectra are fit with white dwarf temperatures near $15,000 \mathrm{~K}$. These temperatures place the accreting white dwarfs outside the instability zone for noninteracting DAV white dwarfs and show that the instability strip is complex for accreting white dwarfs.
\end{abstract}

Subject headings: novae, cataclysmic variables — stars: individual (SDSS J013132.39-090122.3,

SDSS J161033.64-010223.3, SDSS J220553.98+115553.7) — stars: oscillations —

ultraviolet: stars

\section{INTRODUCTION}

Since the discovery of the first pulsating white dwarf in an accreting close binary cataclysmic variable (GW Lib; Warner \& van Zyl 1998; van Zyl et al. 2000, 2004), photometry of systems with similar orbital periods and optical spectra has revealed at least 10 more (Warner \& Woudt 2004; Woudt \& Warner 2004; Araujo-Betancor et al. 2005; Vanlandingham et al. 2005; Patterson et al. 2005a, 2005b; Mukadam et al. 2007; Gänsicke et al. 2006; Nilsson et al. 2006). Seven of these pulsating accreting white dwarfs were found by follow-up work on cataclysmic variables $(\mathrm{CVs})$ that were discovered in the Sloan Digital Sky Survey (SDSS; York et al. 2000) by Szkody et al. (2002b, 2003, 2004, $2005,2006)$. The optical clue for candidate pulsating white dwarfs is the presence of broad absorption lines (originating from the white dwarf) flanking the Balmer emission lines (originating from the disk). In order to be able to view the white dwarf, the accretion disk contribution to the optical light has to be small, a situation that occurs at very low mass transfer rates and hence short orbital periods (near 80 minutes). Since the SDSS provides spectra that are about 2 mag fainter than previous surveys, up to $25 \%$ of SDSS-discovered CVs show the white dwarf presence (Szkody et al. 2005). As the analysis of pulsation frequencies can lead to mass determination and other aspects of the internal structure of

\footnotetext{
${ }^{1}$ Based on observations made with the NASA/ESA Hubble Space Telescope, obtained at the Space Telescope Science Institute, which is operated by the Association of Universities for Research in Astronomy, Inc., under NASA contract NAS 5-26555; with the Apache Point Observatory $3.5 \mathrm{~m}$ telescope, which is owned and operated by the Astrophysical Research Consortium; and with the Nordic Optical Telescope, operated on the island of La Palma jointly by Denmark, Finland, Iceland, Norway, and Sweden, in the Spanish Observatorio del Roque de los Muchachos of the Instiitute de Astrofisica de Canaries.

2 Department of Astronomy, University of Washington, Seattle, WA 98195.

3 Department of Physics, University of Warwick, Coventry CV4 7AL, UK.

${ }^{4}$ Department of Astronomy, University of Cape Town, Private Bag, Rondebosch 7700 , South Africa.

5 Institute of Theoretical Astrophysics, Blindern, N-0315 Oslo, Norway.

${ }^{6}$ Gemini Observatory, Hilo, HI 96720; and Subaru Telescope, Hilo, HI 96720.

7 Department of Astronomy and Astrophysics, Villanova University, Villanova, PA 19085.

8 Indian Institute of Astrophysics, Koramangala, Bangalore 560 034, India.

9 American Association of Variable Star Observers, Cambridge, MA 02138.
}

the white dwarf via the technique of asteroseismology, the compilation of a significant number of pulsating CVs provides a valuable database for probing the effects of mass transfer and accretion on the long-term evolution of white dwarfs as well as the effect of external heat input, He-enriched envelopes, and fast rotation on the location of the instability strip.

Noninteracting hydrogen atmosphere (DA) pulsating white dwarfs (DAVs or ZZ Ceti stars) show typical nonradial $g$-mode pulsations with periods of 50-1400 s and have temperatures in the narrow range of 10,800-12,300 K and $\log g \sim 8$ (Bergeron et al. 2004; Koester \& Allard 2000; Mukadam et al. 2004; Gianninas et al. 2005). While the pulsation frequencies and amplitudes of CV pulsators appear to be similar to those of ZZ Ceti systems (van Zyl et al. 2004; Warner \& Woudt 2004; Woudt \& Warner 2004; Mukadam et al. 2007), the temperatures of the underlying white dwarfs may be quite different. UV observation of GW Lib (Szkody et al. 2002a) with the Space Telescope Imaging Spectrograph (STIS) revealed a white dwarf with a temperature of $14,700 \mathrm{~K}$ (or a best fit with $63 \%$ of the white dwarf having a temperature of $13,300 \mathrm{~K}$ and the remainder at $17,100 \mathrm{~K}$ ), temperatures far outside the empirical instability strip for noninteracting ZZ Ceti stars. Townsley et al. (2004) could match the pulsation periods and high temperature if they assumed $\ell=1 \mathrm{~g}$-modes and a $1 M_{\odot}$ white dwarf with an accreted H-rich layer of $3 \times 10^{-5} M_{\odot}$. On the other hand, Araujo-Betancor et al. (2005) used a snapshot STIS spectrum to show that the CV pulsator HS 2331+3905 could be fit with a $10,500 \mathrm{~K}, 0.6 M_{\odot}$ white dwarf, within the range of normal ZZ Ceti stars. Of course, pulsating CV white dwarfs are rotating very rapidly compared to noninteracting ZZ Ceti stars, and the thermal profiles are quite different because the surface layer is heated from above by compression and irradiation due to accretion. Although the accretion rates for the CV pulsating white dwarfs are small $\left(\sim 10^{-13} M_{\odot} \mathrm{yr}^{-1}\right)$, the thermal profiles may still be altered, and the effective temperature might not be the best parameter to locate the instability strip. This does not imply that the temperature in the driving regions is not important in locating the instability strip, but only that the effective temperature may not be reflecting the temperature in the driving regions due to accretion heating in the surface layers. Arras et al. (2006) show that the temperature in the driving zone, $\log g$, and the He abundance are all important for accreting white dwarfs. 
TABLE 1

Source Characteristics

\begin{tabular}{|c|c|c|c|c|c|}
\hline SDSS J & $\begin{array}{c}g \\
(\mathrm{mag})\end{array}$ & $\begin{array}{c}P_{\text {orb }} \\
\text { (minutes) }\end{array}$ & $\begin{array}{l}\text { Optical Pulsation Period } \\
\text { (s) }\end{array}$ & $\begin{array}{l}\text { Amplitude } \\
\text { (mmag) }\end{array}$ & References \\
\hline $0131 \ldots \ldots \ldots \ldots \ldots \ldots \ldots \ldots \ldots$ & 18 & 98 & $260,335,595$ & $4,9,17$ & Warner \& Woudt (2004) \\
\hline 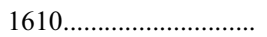 & 19 & 81 & $345,607^{\mathrm{a}}$ & 7,27 & Woudt \& Warner (2004) \\
\hline $2205 \ldots \ldots \ldots \ldots \ldots \ldots \ldots \ldots \ldots \ldots \ldots \ldots \ldots \ldots \ldots \ldots$ & 20 & $\ldots$ & $330,475,575$ & $10,7,8$ & Warner \& Woudt (2004) \\
\hline
\end{tabular}

a Several combinations of these two independent periods are also evident.

In order to explore the physical parameter space occupied by accreting pulsators (Arras et al. 2006), and to attempt mode identification through comparison of UV to optical amplitudes (Robinson et al. 1995), we proposed further study of three additional known pulsators with STIS. After the proposal was accepted, STIS was taken out of service, but we were able to switch our program to the Solar Blind Channel (SBC), which has the same wavelength coverage, albeit much lower resolution and no TIMETAG mode of photon counting. The three objects are SDSS J013132.39-090122.3 (Szkody et al. 2003; Warner \& Woudt 2004), SDSS J161033.64-010223.3 (Szkody et al. 2002b; Woudt \& Warner 2004), and SDSS J220553.98+115553.7 (Szkody et al. 2003; Warner \& Woudt 2004). Throughout the rest of this paper, we refer to these objects as SDSS J0131, SDSS J1610, and SDSS J2205, respectively. A summary of the orbital periods, visual brightness, and optical pulsation periods is provided for these three systems in Table 1. The SDSS spectra are shown in Figure 1.

\section{OBSERVATIONS AND REDUCTIONS}

\subsection{HST Ultraviolet Observations}

The Hubble Space Telescope (HST) UV data were acquired using the prism PR110L and the Solar Blind Channel on the Advanced Camera for Surveys (ACS) to provide UV spectra from approximately 1245 to $1800 \AA$. Due to the prism, the dispersion varied from about $1.5 \AA \mathrm{pixel}^{-1}$ at the far UV end to about $25 \AA \mathrm{pixel}^{-1}$ at the longest wavelengths. Each system was observed for five $H S T$

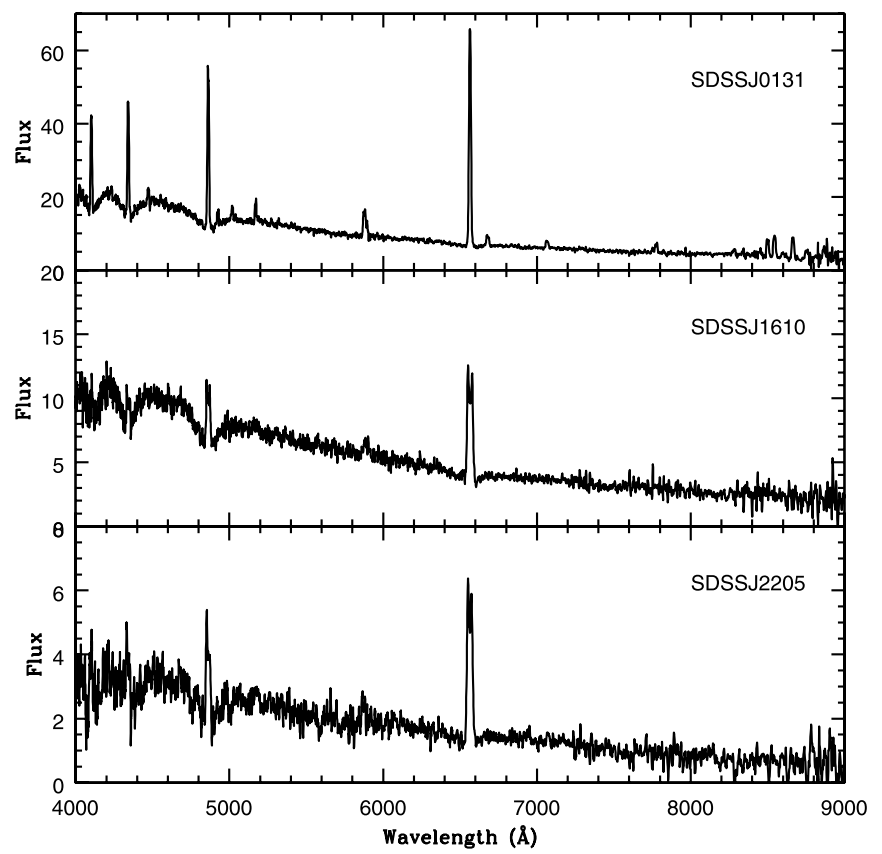

FIG. 1.- SDSS spectra of our three sources, showing the absorption troughs surrounding the Balmer emission lines. Units of flux are $10^{-17} \mathrm{ergs} \mathrm{cm}^{-2} \mathrm{~s}^{-1} \AA^{-1}$. orbits using $61 \mathrm{~s}$ integrations in ACCUM mode. The dead time between observations was $40 \mathrm{~s}$, so the time resolution of the spectra are $101 \mathrm{~s}$. The first orbit had 26 integrations (due to the initial overhead of setting up on a target), and the remaining four orbits contained 29 integrations. Thus, the total time on each source consisted of 142 integrations of $61 \mathrm{~s}$ each, or $8662 \mathrm{~s}$.

The UV data were analyzed using tools available under STSDAS and PyRAF (aXe14). The primary target was extracted using a variety of extraction widths to optimize the signal-to-noise ratio $(\mathrm{S} / \mathrm{N})$ of the resulting spectra and light curves. For the spectra, a wide extraction of 17 pixels was used, and the resulting 142 spectra were co-added to produce a final spectrum. For the light curves, a smaller extraction width produced the optimum (largest) pulse signal. This optimum extraction width was determined to be 9 , 12, and 4 pixels for SDSS J0131, J1610, and J2205, respectively. The fluxes were then added across wavelength for each individual spectrum to produce photometric points throughout the five orbits for a light curve that could be analyzed for periodicity using discrete Fourier transforms. For the two brightest systems (SDSS J0131 and J1610), the spectra at the peaks and troughs of the resulting light curves were then individually combined into peak and trough summed spectra, as any difference in temperature between these spectra would help constrain the change in temperature during a pulsation cycle of the dominant mode.

\subsection{Optical Observations}

In order to prevent excessive UV light from entering the ACS detectors, STScI required continual ground observations prior to and during the HST observations, to ensure that the objects were not going into a dwarf nova outburst. These monitoring observations were kindly provided by a large group of worldwide amateurs and professionals (from the American Association of Variable Star Observers [AAVSO] and the Robotic Optical Transient Search Experiment [ROTSE] and scientists at observatories for other programs). All three systems remained at quiesence throughout the planning and execution of the observations.

Coordinated simultaneous optical observations were achieved for SDSS J1610 in order to obtain the amplitude and period of the optical pulsations. The $3.5 \mathrm{~m}$ telescope at Apache Point Observatory (APO) was used with a $B$ filter on the CCD camera SPIcam, along with the Nordic Optical Telescope (NOT) $2.56 \mathrm{~m}$ telescope and $B$ filter on the Faint Object Spectrograph and Camera (ALFOSC) on the night of June 30/July 1 . APO used an integration time of $20 \mathrm{~s}$, while NOT used $14.1 \mathrm{~s}$. Additional data were obtained on the $2 \mathrm{~m}$ Himalayan Chandra Telescope (HCT) on the nights preceding and following the HST observation. These observations were accomplished with no filter and with $30 \mathrm{~s}$ integration times.

To check the stability of periods for SDSS J0131, additional APO data were obtained on 2005 December 1 and 5 and also on 2006 January 30, using the imaging CCD on the Dual Imaging Spectrograph (DIS) with no filters. DIS utilizes a dichroic that splits the light at $5550 \AA$, so that the blue and red portions of the 
TABLE 2

Summary of Observations

\begin{tabular}{|c|c|c|c|c|c|}
\hline SDSS $J$ & Observed with & Instrument & Filter & $\begin{array}{l}\text { Interval } \\
\text { (s) }\end{array}$ & Time \\
\hline $0131 \ldots \ldots \ldots . . .$. & $H S T$ & SBC & PR110L & 61 & 2005 Jun 18 1:35:49-8:42:35 \\
\hline $0131 \ldots \ldots \ldots . . . .$. & APO & DIS & none & 15,30 & 2005 Dec 05 07:25:21-08:19:53 \\
\hline $0131 \ldots \ldots \ldots \ldots$ & APO & DIS & none & 15 & 2006 Jan 30 01:41:23-03:12:27 \\
\hline $0131 \ldots \ldots \ldots \ldots$ & SAAO & UCTCCD & none & 30 & $2003 \mathrm{Jul} 2(2.18 \mathrm{hr}), 3(2.23 \mathrm{hr}), 5$ (2.17 hr) \\
\hline $0131 \ldots \ldots \ldots \ldots$ & SAAO & UCTCCD & none & 120,100 & $2003 \mathrm{Jul} 25(3.34 \mathrm{hr}), 28(3.47 \mathrm{hr})$ \\
\hline $0131 \ldots \ldots \ldots \ldots$ & SAAO & UCTCCD & none & 60 & 2004 Sep $14(2.88 \mathrm{hr}), 15(3.48 \mathrm{hr}), 16(5.10 \mathrm{hr}), 19(3.13 \mathrm{hr}), 20(5.11 \mathrm{hr}), 21(0.83 \mathrm{hr})$ \\
\hline $0131 \ldots \ldots \ldots \ldots$ & SAAO & UCTCCD & none & $30-60$ & 2004 Nov $3(5.43 \mathrm{hr}), 4(1.53 \mathrm{hr}), 5(3.21 \mathrm{hr}), 6(4.57 \mathrm{hr})$ \\
\hline $0131 \ldots \ldots \ldots . . . .$. & SAAO & UCTCCD & none & 20 & 2006 Aug 25 (4.03 hr), 26 (4.78 hr), $27(5.05 \mathrm{hr})$ \\
\hline $1610 \ldots \ldots \ldots \ldots$ & $\mathrm{HCT}$ & $\mathrm{CCD}$ & none & 30 & 2005 Jun 30 15:21:21-16:22:56 \\
\hline $1610 \ldots \ldots \ldots \ldots$ & $H S T$ & SBC & PR110L & 61 & 2005 Jun 30 23:13:19-2005 Jul 01 6:18:13 \\
\hline $1610 \ldots \ldots \ldots \ldots$ & NOT & ALFOSC & $\mathrm{B}$ & 14.1 & 2005 Jun 30 22:16:59-2005 Jul 01 03:29:39 \\
\hline
\end{tabular}

beam are then incident on two distinct CCD cameras. The light curves obtained using the blue CCD camera are used in this paper, sensitive in the wavelength range $3500-5550 \AA$. Windowing was used to read a small portion of the CCD in order to reduce the readout time and obtain a suitable time resolution. A standard IRAF ${ }^{10}$ reduction to extract sky-subtracted light curves from the CCD frames using weighted circular aperture photometry (O'Donoghue et al. 2000) was employed. The optical light curve of the target star was divided by a sum of one or more brighter comparison stars and converted to the same fractional amplitude scale and the times converted to Barycentric Coordinated Time (Standish 1998). A discrete Fourier transform (DFT) was then computed for all the optical light curves up to the Nyquist frequency.

Additional optical data for SDSS J0131 over a longer timescale were obtained from the $1.9 \mathrm{~m}$ Radcliffe telescope at the Sutherland site of the South African Astronomical Observatory (SAAO), using the University of Cape Town CCD Photometer (UCTCCD; O’Donoghue 1995) with no filter. Data were acquired during several nights in 2003 July, August, and September; 2004 September and November; and 2006 August. Fourier transforms (FTs) were computed for each night of data, as well as combined FTs for each month.

10 IRAF (Image Reduction and Analysis Facility) is distributed by the National Optical Astronomy Observatories, which are operated by AURA, Inc., under cooperative agreement with the National Science Foundation.
A summary of the HST and ground-based observations is given in Table 2.

\section{LIGHT CURVES AND PULSATIONS}

The summed photometry from each HST spectrum was analyzed in a similar manner as the DIS data. Figure 2 shows a light curve for a single HST orbit for each system; the pulsation is apparent in each case. The combined data from all five orbits were then analyzed with DFT routines to obtain the quantitative periods and amplitudes. Figure 3 shows the amplitude plots and Table 3 summarizes the resulting periods and amplitudes. The APO, NOT, and HCT data were analyzed in the same way and the results shown in Table 3 and Figure 4.

The optimum data set exists for SDSS J1610, since we can compare periods and amplitudes from simultaneous UV and optical data observations. Figure 2 shows that the UV pulse is most clearly resolved for this system. Table 3 shows that the observed UV and optical periods are identical, while the UV amplitudes are increased by $2-6$ times over those of the optical. Our optical results are consistent in period and amplitude with the two independent periods that were evident in the past data of Woudt \& Warner (2004), as listed in Table 1. The observed $304 \mathrm{~s}$ period is a harmonic of the $608 \mathrm{~s}$ period.

For pulsating white dwarfs, the identification of the excited nonradial $g$-modes can provide the total mass of the star, as well as the mass of the H layer (Kawaler \& Hansen 1989). Each of the eigenmodes that can be excited in the star is described by a set

TABLE 3

Summary of Observed Periods (HST, APO, NOT, HCT)

\begin{tabular}{|c|c|c|c|}
\hline SDSS $J$ & $\begin{array}{l}\text { Wavelength } \\
\text { (§̊) }\end{array}$ & $\begin{array}{l}\text { Period } \\
\text { (s) }\end{array}$ & $\begin{array}{l}\text { Amplitude } \\
\text { (mmag) }\end{array}$ \\
\hline 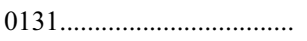 & $1255-1800$ & $213.72 \pm 0.10$ & $78.0 \pm 8.8$ \\
\hline 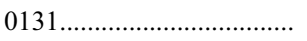 & optical & $581.3 \pm 3.8 ; 211.8 \pm 1.2 ; 79.3 \pm 0.2$ & $31.3 \pm 3.3 ; 13.6 \pm 3.3 ; 9.5 \pm 3.3$ \\
\hline 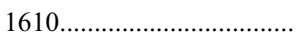 & $1245-1800$ & $608.22 \pm 0.30 ; 304.10 \pm 0.29^{\mathrm{a}} ; 220.81 \pm 0.31$ & $186.1 \pm 7.5 ; 48.3 \pm 7.5 ; 23.4 \pm 7.4$ \\
\hline 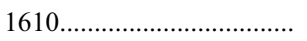 & optical & $608.20 \pm 0.28 ; 304.16 \pm 0.20^{\mathrm{a}} ; 220.68 \pm 0.09$ & $30.6 \pm 2.2 ; 10.5 \pm 2.2 ; 12.2 \pm 2.2$ \\
\hline 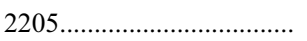 & $1202-1800$ & $576.2 \pm 1.6$ & $46 \pm 11$ \\
\hline
\end{tabular}

a $304 \mathrm{~s}$ period is a harmonic of the $608 \mathrm{~s}$ period. 


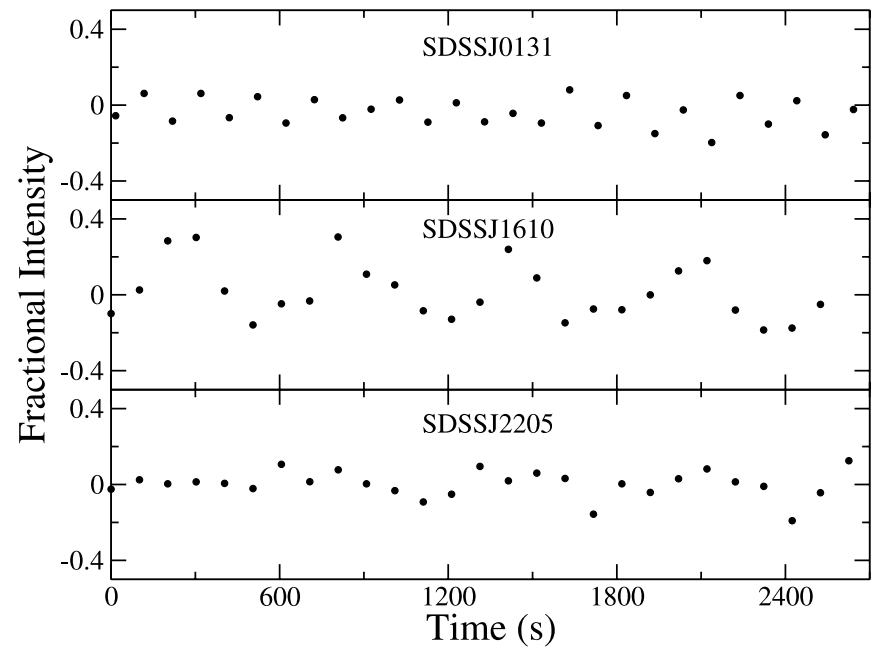

FIG. 2.- Single-orbit SBC light curves from the extracted spectra for each of our three systems. Each point is one $61 \mathrm{~s}$ integration.

of indices: $k$ is the radial quantum number that gives the number of nodes between the surface and the center of the star, $\ell$ is the azimuthal quantum number that gives the number of nodes on the surface, and $m$ is the number of nodes along the meridian, which is used to describe the frequency if the spherical symmetry is lost due to rotation or a magnetic field. We use spherical harmonics $\left(\mathrm{Y}_{m}^{\ell}\right)$ to describe these eigenmodes. This identification of the quantum numbers $k, \ell$, and $m$ for each observed period is not easy, as it involves matching observed periods with predictions from models (with many free parameters). Robinson et al. (1995) pioneered a method of determining the $\ell$-value based on the change in amplitude as a function of wavelength due to limb darkening and the modified geometric cancellation (Robinson et al. 1982). Limb darkening effectively reduces the viewing area of the stellar disk, and this reduction in area depends on wavelength. At UV wavelengths, the increased limb darkening decreases the contribution of zones near the limb, and modes of $\ell=3$ are canceled less effectively in the UV compared to $\ell=1$ or 2 . However, this does not hold true for $\ell=4$ modes, which do not show a significant change in amplitude as a function of wavelength.

Robinson et al. (1995), Nitta et al. (2000), and Kepler et al. (2000) calculated amplitudes in the UV for different values of $\ell$. To attempt to constrain the $\ell$-value for the $608 \mathrm{~s}$ period observed in SDSS J1610, we determined the amplitudes for this period for four different wavelength regions: $1245-1360 \AA, 1360-1500 \AA$, 1500-1640 $\AA$, and 1640-1800 $\AA$. The effective wavelengths of these regions were computed using the best-fit white dwarf model (§ 4). We also computed the amplitudes for 20 individual wavelength bins, but the resulting error bars were too large to be useful. Figure 5 shows the observed pulse amplitude ratios for our four wavelength regions along with the theoretical amplitude ratio for single DA white dwarf stars using Koester's atmosphere models (Finley et al. 1997). The top panel shows the model calculations for $\ell=1-4$ modes for $\log g=8, T_{\text {eff }}=14,500 \mathrm{~K}$ (top) and $12,500 \mathrm{~K}$ (bottom). While neither plot can fit all the wavelengths, the $\ell=1$ mode with $T_{\text {eff }}=12,500 \mathrm{~K}$ is a good fit to all but the shortest wavelength, and it appears that high-order modes ( $\ell=3$ and 4$)$ can be ruled out. However, since the temperatures for accreting white dwarfs are quite different than for DAVs (see below), the simple noninteracting DA model may not be applicable. In addition, since the disk may cast a shadow on the equatorial regions of the white dwarf, the limb darkening and geometric cancellation may be different than that for single white dwarfs.

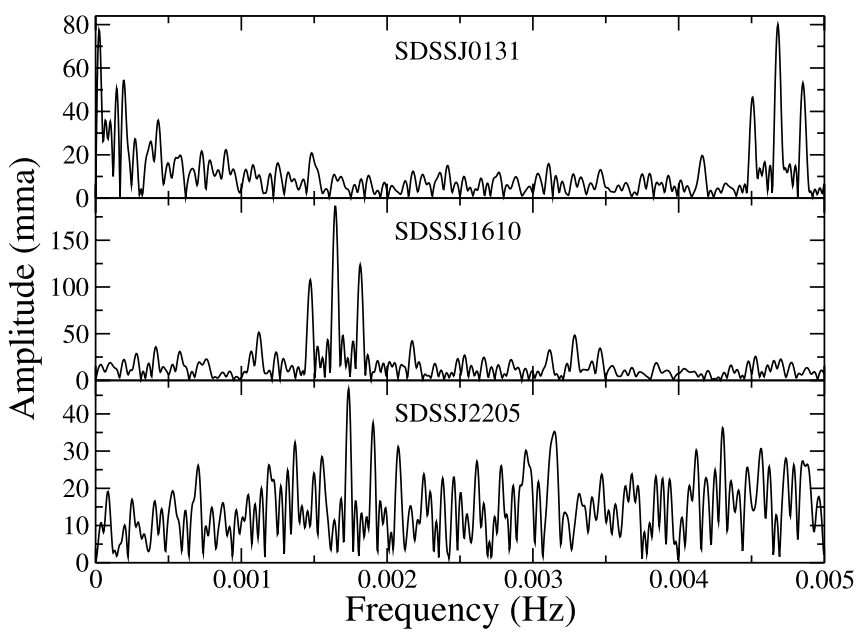

FIG. 3.-DFTs of the combined light curves for the five HST orbits of each system.

It is also interesting that the peaks in the FT of SDSS J1610 show no splitting (Fig. 4), even though the resolution is $\sim 10^{-5} \mathrm{~Hz}$ ( $v \sin i \sim 0.5 \mathrm{~km} \mathrm{~s}^{-1}$ ), whereas fitting of line widths in HST spectra have shown velocities of about $50-100 \mathrm{~km} \mathrm{~s}^{-1}$ (Sion et al. 1994) for accreting white dwarfs. However, there may be some process that is suppressing some of the modes. Long-term monitoring of GW Lib (van Zyl et al. 2004) showed clusters of periods, separated by only about $1 \mu \mathrm{Hz}$, whose origin is not clear. It will take a much longer combined run on SDSS J1610 to tell if this is also the case in this system.

For the other two objects, where simultaneous optical light curves could not be obtained, we can only compare the observed UV periods and amplitudes with available optical values. For SDSS J2205, the single observed UV period of $576 \mathrm{~s}$ matches one of the previously observed optical periods noted by Warner \& Woudt (2004), and the UV amplitude is about 6 times the optical value. The strongest period (330 s) observed in the optical by Warner \& Woudt is not visible in the UV.

The brightest system (SDSS J0131) shows no UV pulsation at the highest amplitude period (595 s) previously evident in the optical data published in Warner \& Woudt (2004). Their other two

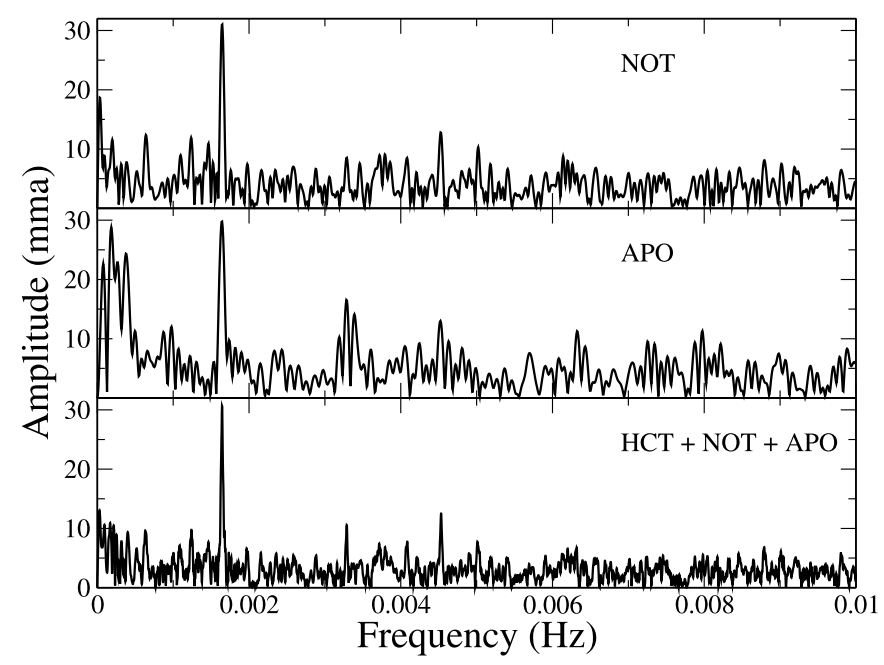

FIG. 4.-DFTs of the APO and NOT data obtained simultaneously with the HST observation of SDSS J1610 and the combined DFT of the APO, NOT, and HCT data. 


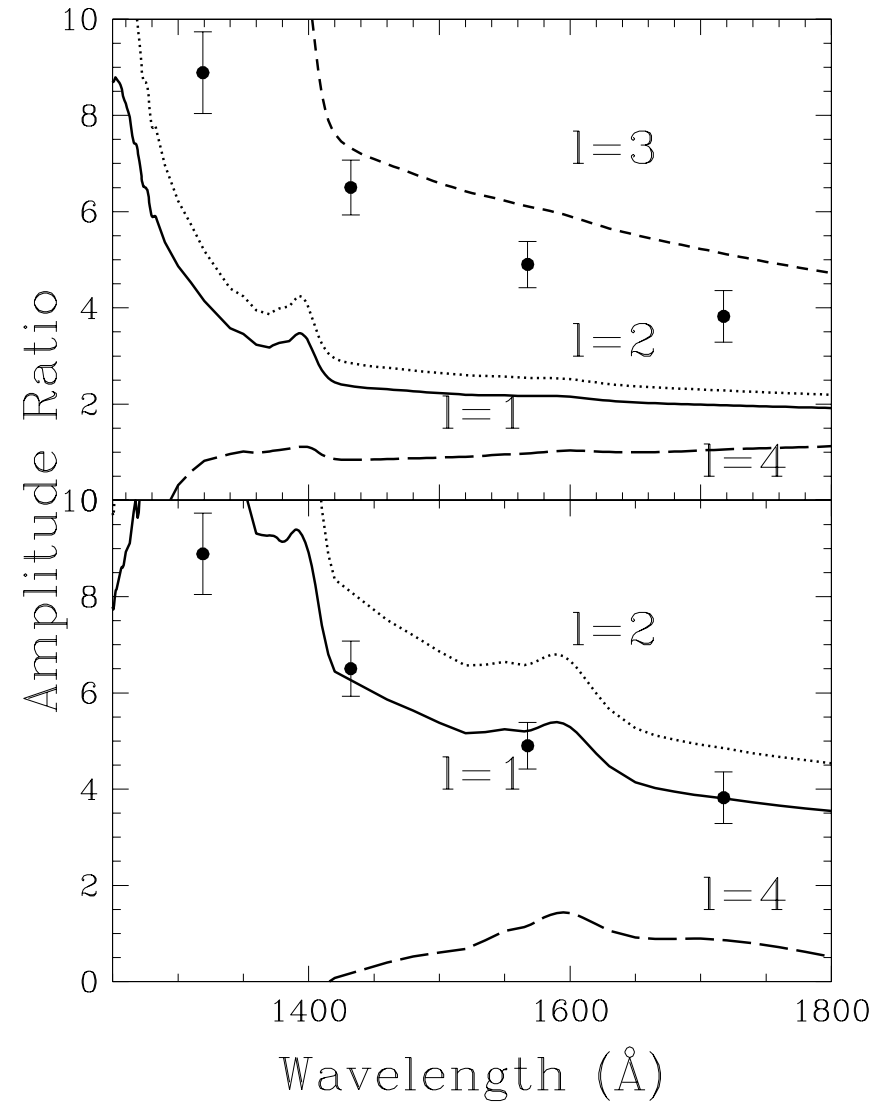

FIG. 5.-Amplitude ratio expected for different modes of the $608 \mathrm{~s}$ pulsation for DA white dwarfs of $\log g=8$ and two different $T_{\text {eff }}: 14,500 \mathrm{~K}$ (top) and $12,500 \mathrm{~K}$ ( bottom). Observed ratios at four different UV wavelengths sampled for SDSS J1610 are superposed.

optical periods (260 and $335 \mathrm{~s}$ ) are also not apparent in the SBC data. Instead, a shorter period of $213 \mathrm{~s}$ near the Nyquist frequency is evident in the $H S T$ data with an amplitude near 80 mmag. The 213 s period is obvious, although just barely resolved, in the SBC light curve (Fig. 2). This is the shortest period yet seen in an accreting pulsator. Our extended optical coverage on this object with APO in the months following the HST observation and with SAAO in the years preceding and following the HST (Table 2) provides a handle on the stability of the observed periods. Figure 6 shows the DFT of the 2006 January data, while Figure 7 shows all the monthly combined runs from the SAAO (Table 4 lists the observed frequencies and periods from the SAAO runs). It is clear that the pulsation spectra are highly variable, with longer periods most prominent in the runs of 2003 (Warner \& Woudt [2004] use data from 2003 September), while the 2004 runs show only the short period seen in the 2005 HST data. Then, in 2006 January (Fig. 6), both the long period (near $600 \mathrm{~s}$ ) and the short (213 s) period are evident, and in 2006 August (Fig. 7), these are both still present, along with an even longer period (1130 s) that is similar to one seen in 2003 August. While changes in pulsation spectra are common in cool noninteracting ZZ Ceti stars on timescales of months and even days, it is not obvious what is to be expected with these hotter pulsators. Long-term monitoring of GW Lib (van Zyl et al. 2004) does show that its pulsation amplitudes are unstable as well. The two periods near 650 and $370 \mathrm{~s}$ remain visible in all runs (including the $H S T$ data), while other periods appear and become invisible in other runs. In SDSS J0131, it appears that all periods show large amplitude variations. Comparing the amplitude of the $213 \mathrm{~s}$ period in the UV with that seen

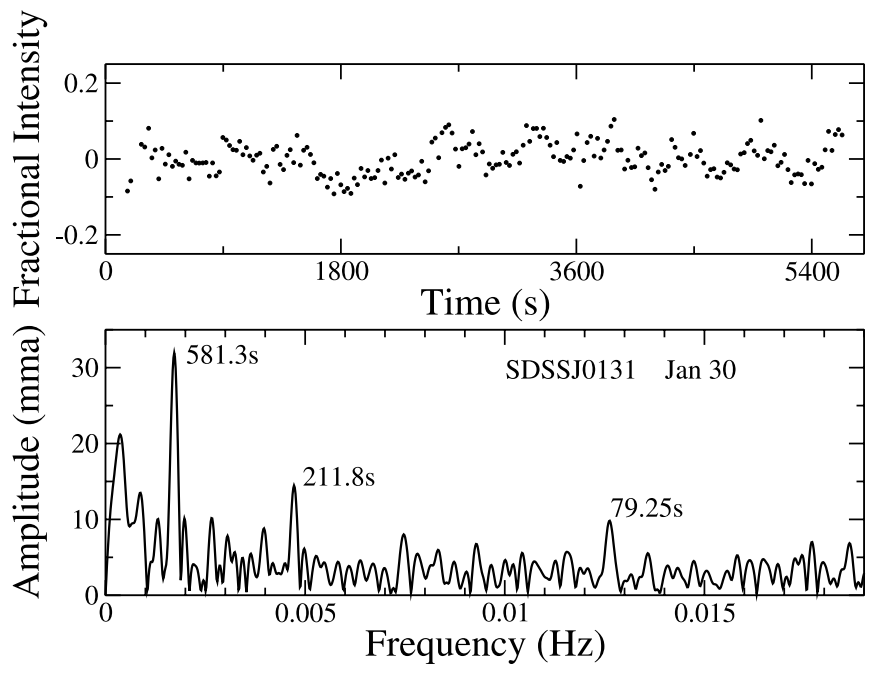

FIG. 6.- Light curve and DFT for 2006 January 30 APO data on SDSS J0131.

in the optical gives a ratio of about 6 , similar to that of SDSS $\mathrm{J} 1610$ and $\mathrm{J} 2205$.

\section{SPECTRAL FITS}

The UV spectra of low mass transfer rate CVs are a combination of the underlying white dwarf, the accretion disk and a source of emission lines (likely an accretion disk chromosphere). To model the observed SBC spectra, we employed a procedure similar to our analysis of STIS data on similar systems (e.g., Gänsicke et al. 2005). This involved using Hubeny white dwarf LTE models TLUSTY195 and SYNSPEC46 (Hubeny \& Lanz 1995 ) in a grid of temperatures, gravities, and abundances to create a best fit to the spectrum. Due to the low resolution of the SBC and the past results, which were all consistent with $\log g=8$, we fixed the $\log g$ at this value and used 0.01 solar abundance to fit the best-temperature white dwarf. The constraints on the fit

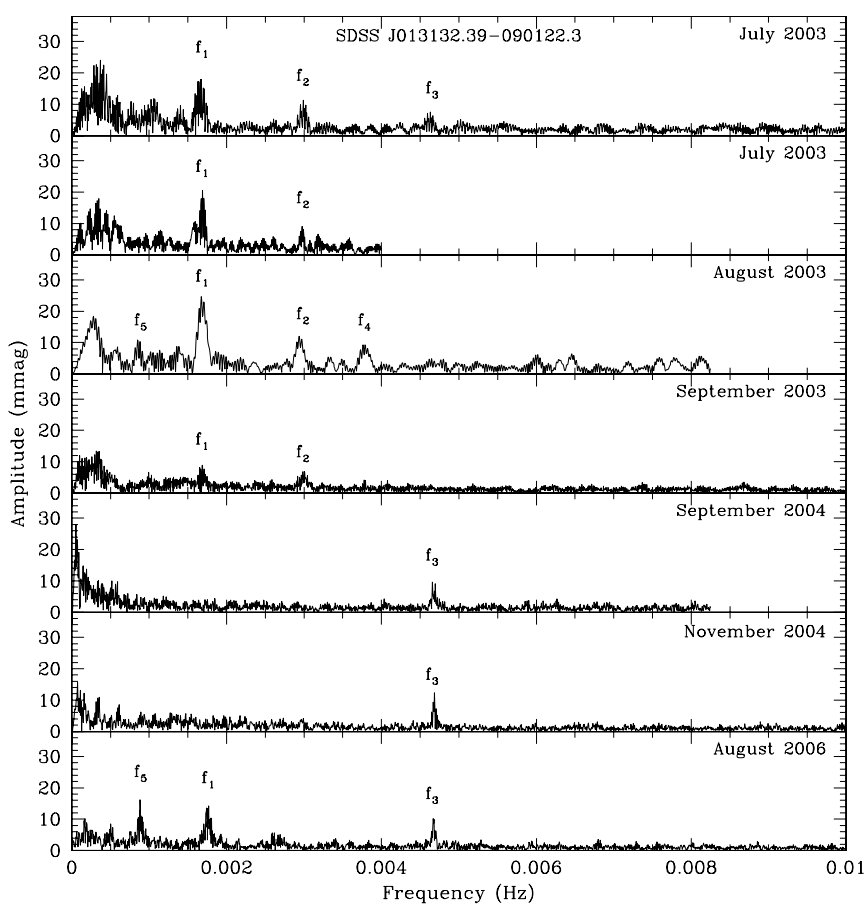

FIG. 7.-FTs for all SAAO data on SDSS J0131. 
TABLE 4

Summary of SAAO Periods For SDSS J0131

\begin{tabular}{|c|c|c|c|c|}
\hline Run & $\begin{array}{c}\text { Frequency } \\
\qquad(\mu \mathrm{Hz})\end{array}$ & $\begin{array}{l}\text { Period } \\
\text { (s) }\end{array}$ & $\begin{array}{c}\text { Amplitude } \\
\text { (mmag) }\end{array}$ & Commen \\
\hline \multirow[t]{3}{*}{2003 Jul $2-5 \ldots \ldots \ldots \ldots . . . .}$. & $1664.1 \pm 0.2$ & 600.9 & $20.9 \pm 2.1$ & $\mathrm{f} 1^{\mathrm{a}}$ \\
\hline & $2989.5 \pm 0.3$ & 334.5 & $12.1 \pm 2.2$ & f2 \\
\hline & $4629.5 \pm 0.4$ & 216.0 & $8.0 \pm 2.2$ & $f 3^{\mathrm{a}}$ \\
\hline \multirow[t]{3}{*}{2003 Jul 25-28.......... } & $1690.0 \pm 0.2$ & 591.7 & $20.6 \pm 3.0$ & $\mathrm{f} 1$ \\
\hline & $1603.1 \pm 0.4$ & 628.8 & $10.4 \pm 3.0$ & \\
\hline & $2970.9 \pm 0.4$ & 336.6 & $9.2 \pm 3.0$ & $\mathrm{f} 2$ \\
\hline \multirow[t]{4}{*}{2003 Aug $20-22 \ldots \ldots}$. & $861.7 \pm 0.4$ & 1160.5 & $11.5 \pm 2.3$ & f5 \\
\hline & $1680.8 \pm 0.2$ & 595.0 & $24.6 \pm 2.4$ & $\mathrm{f} 1$ \\
\hline & $2946.2 \pm 0.4$ & 339.4 & $12.2 \pm 2.3$ & $\mathrm{f} 2$ \\
\hline & $3777.8 \pm 0.5$ & 264.7 & $9.9 \pm 2.4$ & $\mathrm{f} 4$ \\
\hline \multirow[t]{4}{*}{2003 Sep $17-25 \ldots \ldots .}$. & $1687.5 \pm 0.1$ & 592.6 & $13.1 \pm 1.1$ & $\mathrm{f} 1$ \\
\hline & $2965.5 \pm 0.1$ & 337.2 & $8.9 \pm 1.1$ & f2 \\
\hline & $3786.2 \pm 0.2$ & 264.1 & $4.1 \pm 1.1$ & $\mathrm{f} 4$ \\
\hline & $7393.0 \pm 0.2$ & 135.3 & $3.8 \pm 1.1$ & \\
\hline 2004 Sep $14-21 \ldots \ldots .$. & $4671.7 \pm 0.1$ & 214.1 & $10.8 \pm 1.8$ & f3 \\
\hline 2004 Nov $3-6 \ldots \ldots \ldots . . .$. & $4685.3 \pm 0.1$ & 213.4 & $12.7 \pm 1.3$ & f 3 \\
\hline \multirow[t]{3}{*}{2006 Aug $25-27 \ldots \ldots}$. & $885.2 \pm 0.2$ & 1129.6 & $16.2 \pm 1.1$ & f5? \\
\hline & $1758.9 \pm 0.2$ & 568.5 & $14.7 \pm 1.1$ & $\mathrm{f} 1$ ? \\
\hline & $4669.2 \pm 0.3$ & 214.2 & $10.8 \pm 1.1$ & $\mathrm{f} 3$ \\
\hline
\end{tabular}

${ }^{\mathrm{a}}$ f3 may be $\mathrm{f} 1+\mathrm{f} 2$ ?; f 1 may be $2 \mathrm{f} 5$ ?.

include that the white dwarf must provide the best match to the UV continuum shape, especially the broad $\operatorname{Ly} \alpha$ line, and its optical flux must fall below the observed optical fluxes. Figure 8 shows the model results for a range of white dwarf temperatures $(10,000-20,000 \mathrm{~K})$ compared to the observed HST and SDSS spectra of the brightest system SDSS J0131. It is immediately clear that the temperature typical of noninteracting ZZ Cet pulsators $(\sim 12,000 \mathrm{~K})$ is too cool to fit the UV spectrum. A temperature of $14,500 \mathrm{~K}$ is derived from the best fit. This temperature white dwarf extrapolated to optical wavelengths produces a $g$-magnitude of 19.2, well below the observed SDSS $g$-magnitude of 18.3. In addition to the white dwarf, there is some continuum source (the disk) that produces about $30 \%$ of the light across the spectrum. This is especially evident in the core of $\operatorname{Ly} \alpha$, which should be zero for a high-gravity white dwarf. As there is no good model of a non-steady state disk at these low mass transfer rates, we added this continuum component as a simple

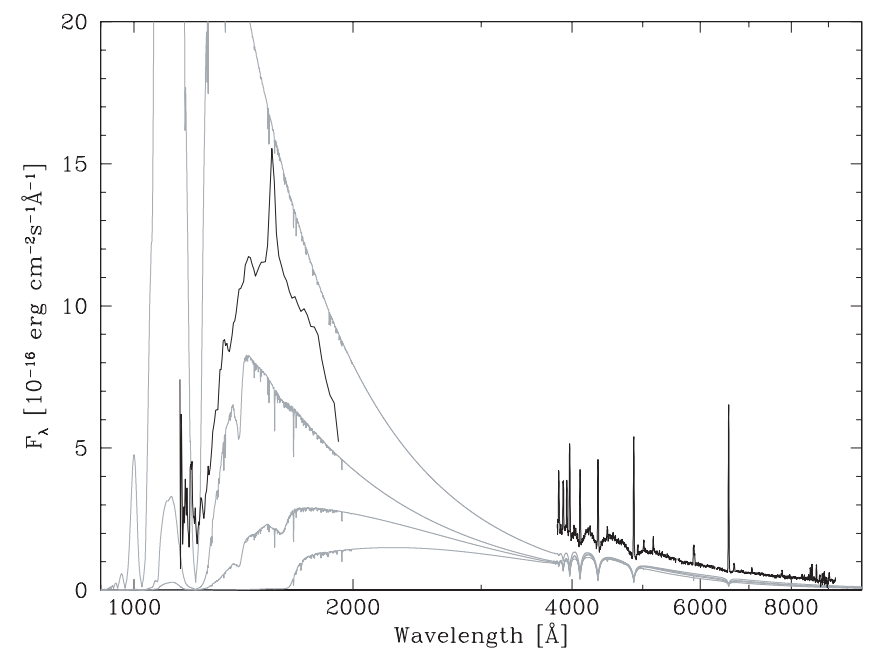

FIG. 8.- SBC and SDSS spectra of SDSS J0131 (black lines) compared with model white dwarfs from 10,000 K, 12,000 K, 14,000 K, and 20,000 K (light gray lines, from bottom to top). The best fit was a $14,000 \mathrm{~K}$ white dwarf.

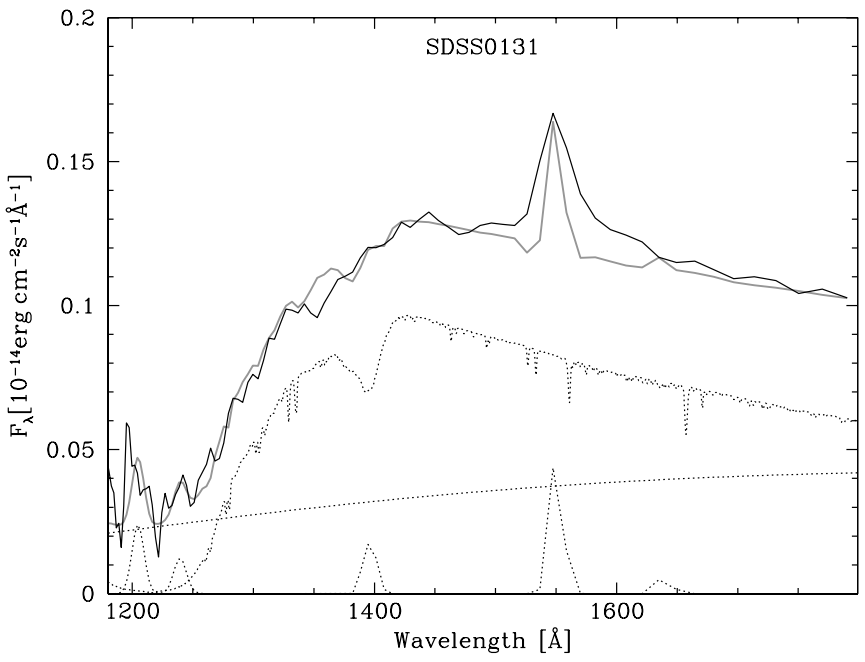

FIG. 9.-Best fit of SBC spectrum of SDSS J0131 with a 14,400 K white dwarf at $350 \mathrm{pc}$ and $15,000 \mathrm{~K}$ blackbody component.

blackbody (power laws were tried with similar results), used simple Gaussians to fill in the emission lines, and redid the fits with the two components. The resulting best fit for SDSS J0131 is shown in Figure 9, and the results are summarized in Table 5. A similar procedure produced the best fits for SDSS J1610 and SDSS J2205 (Figs. 10 and 11 and Table 5).

The spectra that produced the largest amplitude pulse were also added and fit with this procedure. These spectra had higher $\mathrm{S} / \mathrm{N}$ but less overall flux. Thus, the shape of the spectrum may be better defined, but the total fluxes are not correct for distances, etc. We also list these temperature fits as $T_{\text {optext }}$ in Table 5 as a measure of the error of our temperature fits.

We also attempted to fit the difference between the peaks of the pulsations and the troughs for the two brightest sources, SDSS J0131 and SDSS J1610. The spectra of the peak points in the light curves were summed to produce one total peak spectrum, and then the troughs were summed to produce a total trough spectrum. The resulting fits to the peak and trough spectra showed no change in the white dwarf temperatures in either case, which is not surprising given that the uncertainties of our fits are on the order of $1000 \mathrm{~K}$.

The temperatures of the white dwarf in all three systems are similar, close to $15,000 \mathrm{~K}$ and much hotter than the temperatures of the DAV pulsators. These temperatures are also very similar to that derived for GW Lib (Szkody et al. 2002a). Because we had used a two-temperature white dwarf (WD) fit for GW Lib, rather than a WD + BB, we redid the fits to the STIS data of GW Lib using the approach here. The result produces a best fit with $T_{\mathrm{WD}}=$ $15,400 \mathrm{~K}$ and $T_{\mathrm{BB}}=12,000 \mathrm{~K}$, but this fit is not as good as our past fit with two white dwarf components of 13,300 and $17,100 \mathrm{~K}$ covering $63 \%$ and $37 \%$, respectively, of the white dwarf surface.

TABLE 5

White DWARF Fits

\begin{tabular}{|c|c|c|c|}
\hline Parameter & SDSS J0131 & SDSS J1610 & SDSS J2205 \\
\hline$T_{\mathrm{WD}}(\mathrm{K})$ & 14500 & 14500 & 15000 \\
\hline$T_{\mathrm{BB}}(\mathrm{K})$ & 15000 & 12000 & 11000 \\
\hline Distance $(\mathrm{pc}) \ldots \ldots \ldots \ldots \ldots$ & 360 & 480 & 810 \\
\hline$g_{\mathrm{WD}}($ model $) \ldots \ldots \ldots \ldots \ldots$ & 19.3 & 19.9 & 21.0 \\
\hline$g_{\mathrm{WD}}(\mathrm{SDSS}) \ldots \ldots \ldots \ldots$ & 18.3 & 19.1 & 20.1 \\
\hline$T_{\mathrm{WD}}($ optext)$\ldots \ldots \ldots \ldots \ldots$ & 14000 & 14000 & 14000 \\
\hline$T_{\mathrm{BB}}($ optext $) \ldots \ldots \ldots \ldots \ldots$ & 16000 & 16000 & 14000 \\
\hline
\end{tabular}




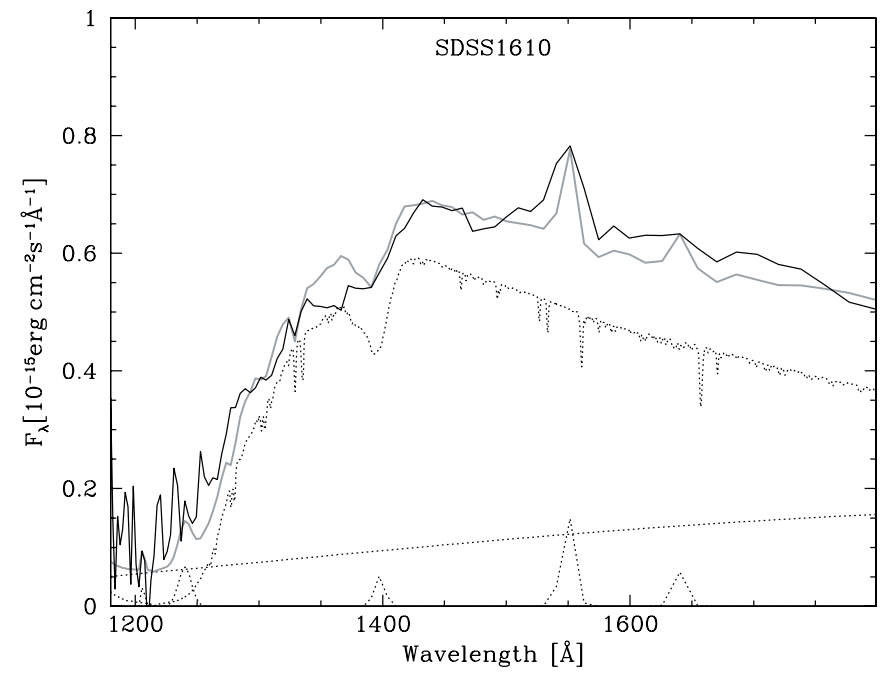

FIG. 10.-Best fit of SDSS J1610 with a $14,500 \mathrm{~K}$ white dwarf at a distance of $480 \mathrm{pc}$ and a $12,000 \mathrm{~K}$ blackbody.

Further confirmation that the two-temperature white dwarf is correct for GW Lib comes from inspection of its STIS spectrum. This shows that the core of Ly $\alpha$ does go down to zero flux, indicating that the white dwarf, rather than a second component such as the disk, contributes all the UV flux. A comparison of the singletemperature white dwarf fit $(14,700 \mathrm{~K})$ with that of the WD+BB also shows that adding in a second component only changes the derived temperature of the white dwarf by less than $1000 \mathrm{~K}$.

Thus, we now have secure results that four of the accreting ZZ Ceti stars in CVs are hotter than single pulsators. On the other hand, HS $2331+3905$ is also secure in having a white dwarf temperature of 10,500 K (see Fig. 13 in Araujo-Betancor et al. 2005). While the UV emission lines in HS $2331+3905$ are much stronger than in the four objects that have hot white dwarfs (likely partly due to the weak white dwarf continuum), and there are continuum variations that might imply a higher inclination, the fact remains that the white dwarf pulsations are visible in HS $2331+3905$, so the white dwarf light is prominent and it is cool. In contrast to our three SDSS sources, all of which have UV fluxes greater than the optical, as in Figure 8, HS 2331 has larger optical than UV fluxes and a distinct rise in flux longward of $1600 \AA$, which pins down the white dwarf temperature to its cool value.

Since accreting white dwarfs are known to show UV absorption lines of $\mathrm{Si}, \mathrm{C}$, and other metals, it is clear they do not have pure $\mathrm{H}$ atmospheres (Sion 1999). Thus, perhaps it is not unexpected that the instability strip is not the same as for ZZ Ceti stars. For accreting model white dwarfs with a high $\mathrm{He}$ abundance $(>0.38)$, Arras et al. (2006) find an additional hotter instability strip at $\sim 15,000 \mathrm{~K}$ due to $\mathrm{He}$ II ionization. They infer that HS $2331+$ 3905 has a low-mass white dwarf, while GW Lib (and hence SDSS J0131, SDSS J1610, and SDSS J2205) should have highly evolved donor stars and more massive white dwarfs.

\section{CONCLUSIONS}

Our low-resolution light curves throughout five HST orbits for our three white dwarf accreting pulsators all have increased pulsation amplitudes in the UV compared to the optical, consistent with $\ell=1$ modes. The simultaneous optical/UV data for SDSS J1610 showed identical periods in both wavelength regions, while SDSS J0131 and SDSS J2205 showed one of three periods evident in the optical at other times. The long-term monitoring of

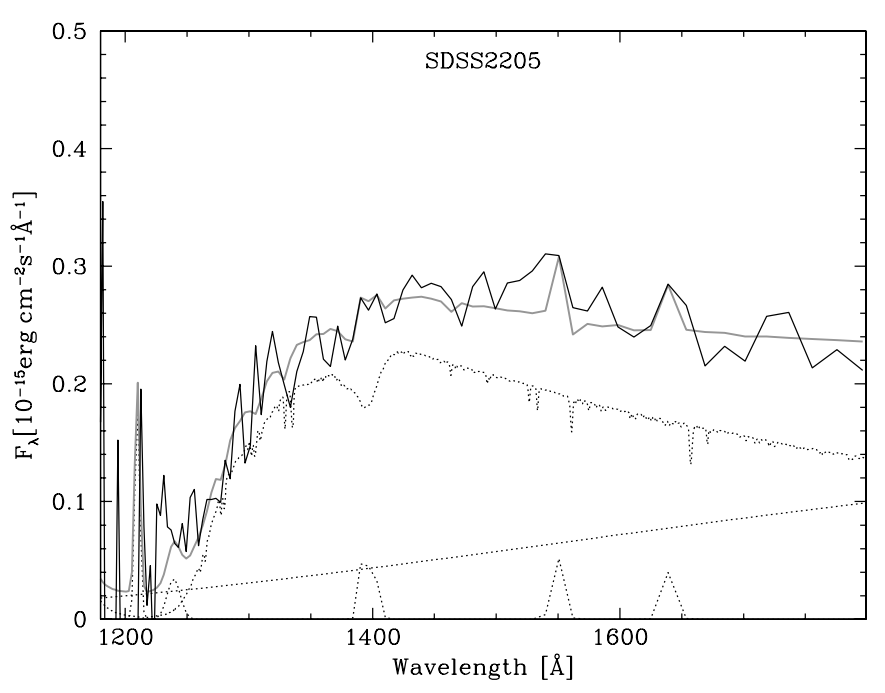

FIG. 11. - Best fit of SDSS J2205 with a 15,000 K white dwarf at a distance of $810 \mathrm{pc}$ and a $11,000 \mathrm{~K}$ blackbody.

SDSS J0131 shows that all pulsation periods come and go, indicating a high level of instability for this system.

The summed UV spectra reveal hot white dwarfs in all three systems, similar to past results on GW Lib but contrary to the lone cool accreting white dwarf pulsator HS 2331+3905. Based on just these five systems with well-determined white dwarf temperatures from the UV, it appears that there is a wide range in the instability strip for accreting systems, with most accreting pulsators in a regime that is hotter than for nonaccreting ZZ Ceti stars. Whether this is due to a difference in the white dwarf mass and/or He composition provided by an evolved donor, as proposed by Arras et al. (2006), can be confirmed if the white dwarf masses and/or the composition of the donor can be determined. As there is no evidence of a donor star out to $9000 \AA$ in the SDSS spectra, and the systems are generally faint, the donor will be difficult to characterize. Since the absorption lines of the white dwarf are difficult to measure in the optical with the contaminating Balmer emission, the best approach may be the use of highresolution UV (the Cosmic Origins Spectrograph) time-resolved data in the future to obtain the mass of the white dwarf, although the unknown inclinations will create some uncertainty.

We are very grateful to the many observers who monitored our objects to insure the safety of the UV detectors and allow our observations to take place, including Ms. Bama, Dick Campbell, Dean Chandler, Katy Garmany (and TLRBSE teachers), Bill Goff, Bernard Heathcote, Arne Henden, Santosh Joshi, Seung-Lee Kim, Mercedes Lopez-Morales, David Mary, John W. McAnally, Aaron Price, Chuck Pullen, ROTSE observers, Patrick Schmeer, and D. E. Winget. We also kindly acknowledge Denise Dale and Ewald Zietsman for the observations of SDSS J0131 taken at the SAAO. This research was supported by NASA grant GO10233.01A and through the Hubble Fellowship grant HST-HF01175.01-A, both awarded from the Space Telescope Science Institute, which is operated by the Association of Universities for Research in Astronomy, Inc., for NASA, under contract NAS 5-26555. B. T. G. was supported by a PPARC Advanced Fellowship. The NOT data were taken using the ALFOSC, which is owned by the Institute de Astrofisica de Andelucia (IAA) and operated by the Nordic Optical Telescope under agreement between IAA and the NBIfAFG of the Astronomical Observatory of Copenhagen. 
Araujo-Betancor, S., et al. 2005, A\&A, 430, 629

Arras, P., Townsley, D. M., \& Bildsten, L. 2006, ApJ, 643, L119

Bergeron, P., Fontaine, G., Billères, M., Boudreault, S., \& Green, E. M. 2004, ApJ, 600, 404

Finley, D. S., Koester, D., \& Basri, G. 1997, ApJ, 488, 375

Gänsicke, B. T., Szkody, P., Howell, S. B., \& Sion, E. M. 2005, ApJ, 629, 451

Gänsicke, B. T., et al. 2006, MNRAS, 365, 969

Gianninas, A., Bergeron, P., \& Fontaine, G. 2005, ApJ, 631, 1100

Hubeny, I., \& Lanz, T. 1995, ApJ, 439, 875

Kawaler, S. D., \& Hansen, C. J. 1989, in IAU Colloq. 114, White Dwarfs, ed. G. Wegner (Berlin: Springer), 97

Kepler, S. O., Robinson, E. L., Koester, D., Clemens, J. C., Nather, R. E \& Jiang, X. J. 2000, ApJ, 539, 379

Koester, D., \& Allard, N. F. 2000, Baltic Astron., 9, 119

Mukadam, A., et al. 2004, ApJ, 612, 1052 2007, ApJ, submitted

Nilsson, R., Uthas, H., Ytre-Eide, M, Solheim, J-E., \& Warner, B. 2006, MNRAS, 370, L56

Nitta, A., et al. 2000, Baltic Astron., 9, 97

O'Donoghue, D. 1995, Baltic Astron., 4, 519

O’Donoghue, D., Kanaan, A., Kleinman, S. J., Krzesinski, J., \& Pritchet, C. 2000, Baltic Astron., 9, 375

Patterson, J., Thorstensen, J. R., Armstrong, E., Henden, A. A., \& Hynes, R. I. 2005a, PASP, 117, 922

Patterson, J., Thorstensen, J. R., \& Kemp, J. 2005b, PASP, 117, 427
REFERENCES

Robinson, E. L., Kepler, S. O., \& Nather, R. E. 1982, ApJ, 259, 219

Robinson, E. L., et al. 1995, ApJ, 438, 908

Sion, E. M. 1999, PASP, 111, 532

Sion, E. M., Long, K. S., Szkody, P., \& Huang, M. 1994, ApJ, 430, L53

Standish, E. M. 1998, A\&A, 336, 381

Szkody, P., Gänsicke, B. T., Howell, S. B., \& Sion, E. M. 2002a, ApJ, 575, L79

Szkody, P., et al. 2002b, AJ, 123, 430

$$
\text { 2003, AJ, 126, } 1499
$$

. 2004, AJ, 128, 1882

2005, AJ, 129, 2386

2006, AJ, 131, 973

Townsley, D. M., Arras, P., \& Bildsten, L. 2004, ApJ, 608, L105

Vanlandingham, K. M., Schwarz, G. J., \& Howell, S. B. 2005, PASP, 117, 928 van Zyl, L., Warner, B., O’Donoghue, D., Sullivan, D., Pritchard, J., \& Kemp, J. 2000, Baltic Astron., 9, 231

van Zyl, L., et al. 2004, MNRAS, 350, 307

Warner, B., \& van Zyl, L. 1998, in IAU Symp. 185, New Eyes to See Inside the Sun and Stars, ed. F.-L. Deubner, J. Christensen-Dalsgaard, \& D. Kurtz (Dordrecht: Kluwer), 321

Warner, B., \& Woudt, P. A. 2004, in IAU Colloq. 193, Variable Stars in the Local Group, ed. D. H. Kurtz \& K. R. Pollard, (ASP Conf. Ser. 310; San Francisco: ASP), 382

Woudt, P. A., \& Warner, B. 2004, MNRAS, 348, 599

York, D. G., et al. 2000, AJ, 120, 1579 